\title{
SCALABLE IMAGE RETRIEVAL FROM DISTRIBUTED IMAGES DATABASE
}

\author{
Tammam Tillo, Marco Grangetto, Gabriella Olmo \\ CERCOM - Center for Multimedia Radio Communications \\ Dipartimento di Elettronica - Politecnico di Torino \\ Corso Duca degli Abruzzi 24 - 10129 Torino - Italy \\ Ph.: +39-011-2276510 - FAX: +39-011-5644099 \\ firstname. secondnameapolito.it
}

\begin{abstract}
In order to store, and retrieve images from large databases, we propose a framework, based on multiple description coding paradigm, that disseminates images over distributed servers. Consequently, decentralized download can be performed, thus reducing links overload and hotspot areas without penalizing downloads speed. Moreover, the tradeoff between system reliability and storage requirement can be achieved by tuning descriptions redundancy, thus providing high flexibility in terms of storage resources, reliability of access, and performance. The scalability of the proposed framework is achieved by the intrinsic progressivity of the multiple description schemes. Moreover, we demonstrate that system can work properly regardless of server crashes.
\end{abstract}

\section{INTRODUCTION}

The technological advances along with the wide spread diffusion of multimedia equipments over the last few years have made it possible to construct large digital image and video libraries comprising tens of tera bytes of online data [1], such as satellite, museums, medical, and seismic-image databases. It is clear that, improving access to such digital libraries creates powerful opportunities for revamping education, accelerating scientific discovery and technical advancement, and improving knowledge[2]. Moreover, due to the continuous growth of image and video data, these libraries will grow significantly larger over the next few years. As a consequence, the demand for the capability to provide databases that can effectively support efficient storage, robustness against failure and transmission of this kind of data will grow significantly.

Moreover, the huge amount of multimedia data causes an increasing interest in those applications that allow to receive data from multiple sources, with neither the

This work has been partially sponsored by MIUR (Italian Ministry of Education and Research) under the project PRIMO - "Reconfigurable platforms for wideband wireless communications" and EU under NewCom Network of Excellence. sources nor the destination users collocated within the same geographical area; this objective needs to develop a wide-area networking that can address the interactive exchange of data among multiple distributed servers and clients, and coordinate multiple data streams destined to a single location [3]. Given the proliferation of internetconnected devices it is become natural to address the internet-based distributed storage system; such a system would let devices transparently share data and preserve information even when devices are lost or damaged [4].

An example of such a system is the Distributed-Parallel Storage System (DPSS) [5], which is a block server that can supply data to applications located anywhere in the network in which it is deployed. In DPSS multiple low-cost, medium-speed disk servers use the network to aggregate their data streams, and information are dispersed in such a way that as many system elements as possible can operate simultaneously to satisfy a given request across both disks and servers. This strategy allows a large collection of disks to be seek in parallel, and all servers to send the resulting data to the application in parallel [5]. Another example of distributed storage system is OceanStore which uses erasure codes and data fragmentation over many servers for data durability [4].

Another application is the TerraVision which allows users to navigate, through a 3-D graphical representation of a real scenery created from images of that area; these data can be distributed over multiple servers across the Web [6], [7].

It is clear that it is important to develop a framework which enable fast access to geographically distributed images over different servers. The information overlap among different servers, should be carefully tuned, so as to achieve the best tradeoff between reliability requirements and hardware costs [8]. Beside the benefit of lowering hardware costs, geographically-dispersed archives allow fast access to information from a wider area.

In [9], the author has highlighted the similarity between multiple description coding (MDC), which is a source 
coding method that exploits the multiple transmission paths between sender and receiver, and the distributed storage framework.

\section{MDC FOR DISTRIBUTED STORAGE}

Image communication systems based on layered coding allow one to reconstruct the transmitted information progressively with a quality level proportional to the amount of received data. A drawback of such a scheme is that any received data segment cannot be exploited unless all previous layers have been received; therefore, in order to exploit all the received information, the base layers must be retransmitted. On the other hand, MDC is a progressive technique where all the transmitted segments of data (descriptions) can be independently decoded and are mutually refinable [9]. Descriptions are created so that the quality of the recovered image is a function of the number of the received descriptions, and not of the specific received pattern. MDC features permit to store the data in a distributed fashion as shown in Fig 1, where two descriptions are placed in geographically separated servers ( $\mathrm{S} 1$ and $\mathrm{S} 2$ ); each client ( $\mathrm{C} 1$ or $\mathrm{C} 2$ ) can access the data from the closest server obtaining a course quality image and decode a full quality image by receiving the complementary description from the other server. The ability to decode any subset of descriptions means that data can be disseminated over a distributed storage system with non-prioritized infrastructure, where all servers share the same level of importance. Moreover, it allows one to distribute the load over all servers avoiding hotspot areas.

Given a certain coding rate, MDC systems performance is evaluated in terms of the quality of the reconstructed signal when decoding all descriptions (central quality) or a subset of the descriptions (side quality). A controllable amount of extra redundancy needs to be inserted between the descriptions so as to minimize the distortion caused by the descriptions that are not available at the decoder. The amount of introduced redundancy represents the storage overhead to be paid for the distributed storage capability and determines a tradeoff between system reliability and storage requirements, which determine system cost.

\section{PRACTICAL MDC FOR DISTRIBUTED STORAGE}

As for actual MDC implementations, plenty of methods have been proposed in the literature. Perhaps the most popular is the class of quantization-based approaches, stemming from the pioneering MD scalar quantization (MDSQ) proposed by Vaishampayan in 1993 [10]. The extension of [10] to the case of more than two descriptions becomes a complicated job. In fact an efficient optimization algorithm able to create the descriptions while controlling the redundancy is not available; the only possibility is to try

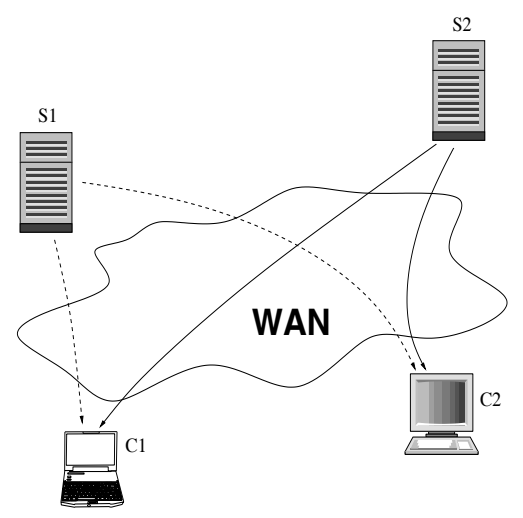

Fig. 1. The generated descriptions are stored in geographically distributed servers, enabling fast access and good tradeoff between reliability and hardware cost.

all possible ways to fill the index assignment hyper-cube [11]. This makes the algorithm impractical for a database of images stored at more than two locations. Another disadvantage of MDSQ is that it is not always possible to generate descriptions with almost no redundancy. On the other hand, rate-distortion MD (RD-MD) algorithm [12] based on post-compression rate allocation codec, such as JPEG 2000, allows to generate $N$-descriptions with the required redundancy.

According to the RD-MD approach, the encoder generates $N$ JPEG 2000 streams using the set of rates $R=$ $\left\{R_{i} ; i=1, . ., N\right\}$ where $R_{1} \geq R_{2} \geq R_{3} \ldots \geq R_{N}$. Then, each description can be generated by properly combining the code blocks (CBs) taken from such bitstreams in order to fulfil the $N$-balanced description paradigm, each description is encoded approximately at $\frac{\sum_{i=1}^{N} R_{i}}{N}$ bps, with a total output rate $R_{t}=\sum_{i=1}^{N} R_{i}$. These descriptions are then stored in the different servers as show in Fig. 1.

At the client side, if more than two descriptions are available, these latter are pre-processed and merged in a single bit stream, where, for each $\mathrm{CB}$, the best representation is selected (the best $\mathrm{CB}$ representation can be easily identified by determining the $\mathrm{CB}$ length); the resulting stream is then JPEG 2000 decoded. On the other hand, if only one description is available, this one is simply JPEG 2000 decoded yielding inferior quality. When a subset of $k$ descriptions out of $N$ are decoded, the distortion can be evaluated as [13]:

$$
D_{k, N}=\frac{k(N-k) !}{N !} \sum_{i=1}^{N-k+1} \frac{(N-i) !}{(N-k-i+1) !} D\left(R_{i}\right)
$$

where $D(r)$ is the RD function of the source data. Finally, given the full compatibility of the generated descriptions with part-1 of the JPEG 2000 standard, a client which is unaware of the distributed storing technique, can still 
retrieve a reduced quality image decoding a single description.

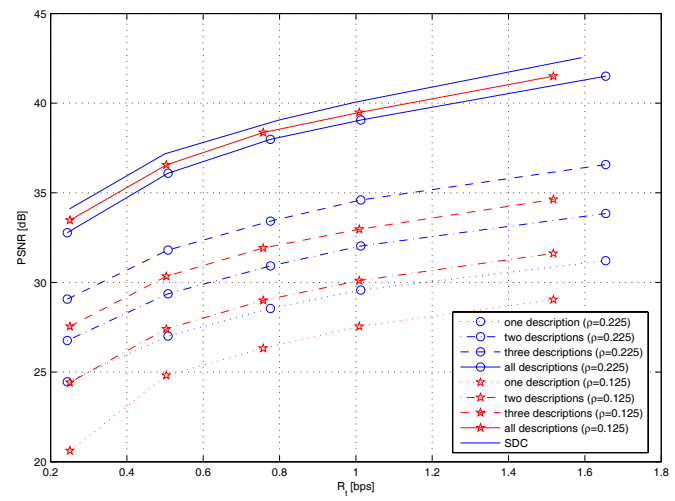

Fig. 2. PSNR vs. total rate (in bps) for image Lenna; SDC and one, two, three and four descriptions reception, with redundancy $\rho=0.225$ and $\rho=0.125$.

\section{EXPERIMENTAL RESULTS}

Simulations have been carried out employing images Lenna and Goldhill of dimension $512 \times 512$, using a modified version of the JPEG 2000 OpenJpeg co-decoder [14]. Four levels of wavelet decomposition are applied with CB of dimension $64 \times 64$. The total output rate is fixed to $0.25,0.50,0.751 .00$ and $1.50 \mathrm{bpp}$ and four balanced descriptions, with redundancy $\rho=0.225$ and $\rho=0.125$, are created. In Fig. 2, 3 we report the peak signal to noise ratios (PSNR) versus the total rate upon decoding 1, 2, 3 and 4 descriptions; moreover we report the single description coding (SDC) performance, which

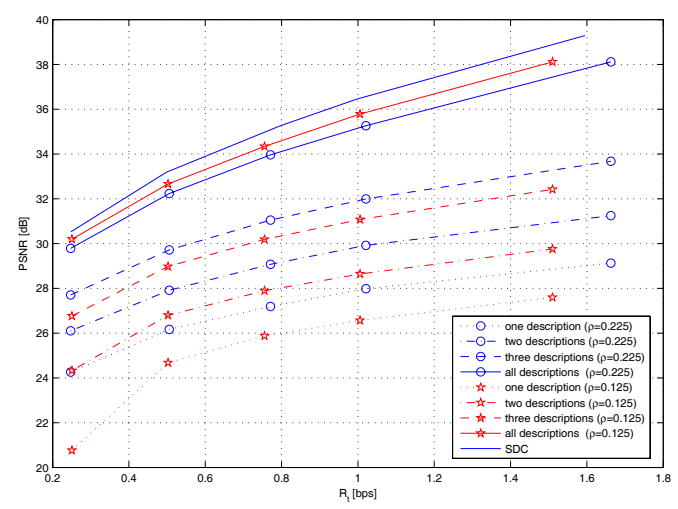

Fig. 3. PSNR vs. total rate (in bps) for image Goldhill; SDC and one, two, three and four descriptions reception, with redundancy $\rho=0.225$ and $\rho=0.125$.

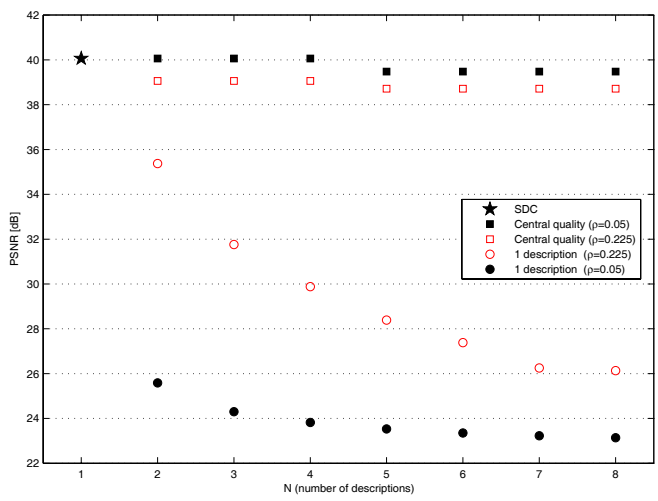

Fig. 4. Central and one description PSNR vs. total number of descriptions for image Lenna encoded at $R_{t}=1 \mathrm{bps}$.

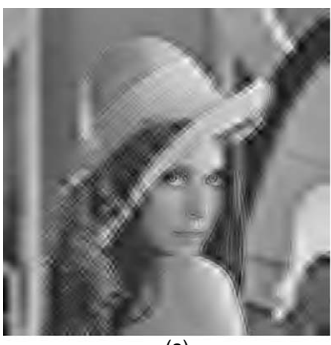

(a)

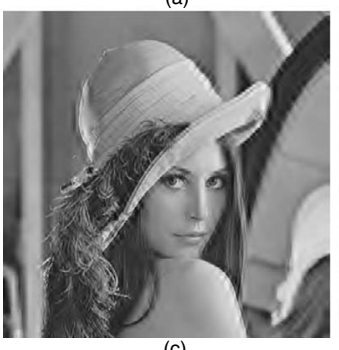

(c)

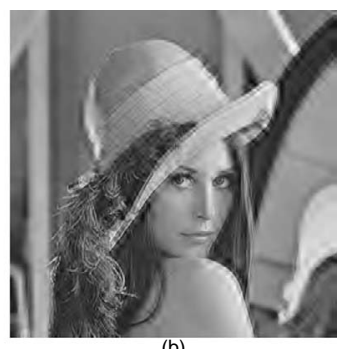

(b)

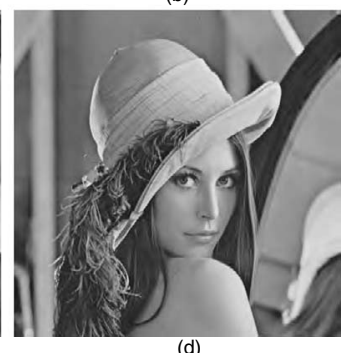

Fig. 5. The obtained image by decoding: a) one; b) two; c) three and d) four descriptions $(N=4)$, of the image Lenna encoded at $R_{t}=1 \mathrm{bps}$ and $\rho=0.05$.

is simply the RD curve of the JPEG2000 encoded image; this modality represents the standard storage case where no data fragmentation is employed. The PSNR is evaluated using the mean square error for all the possible reception patterns. We can notice that, receiving one description, still guarantee acceptable quality and as more descriptions are decoded the quality improves by nearly $3 \mathrm{~dB}$. The impairment due to the inserted redundancy is nearly $1 \mathrm{~dB}$ for $\rho=0.225$ and $0.5 \mathrm{~dB}$ for $\rho=0.125$. This means, that a distributed storage system with MDC paradigm can still guarantees a decodeable bit stream, with acceptable quality, with just one working server out of $N$. On the other hand, the erasure codes-based techniques, like that used in 
OceanStore [4] requires the identical duplication of data in order to guarantee system durability when receiving $1 / N$ of the information, which results in no advantage when receiving multiple copies.

From Fig. 2, 3 we can notice that small changes in the central quality would cause a large change in the side qualities. This is mainly due to the fact that the RD function are convex, and consequently when working at fixed total rate small changes in redundancy causes large changes in side performance.

It is important to tune the inserted redundancy, in order to get the best tradeoff between reliability and storage constraints. In the limit case where server storage capacity allows to store duplicated copy of the images over all servers, it becomes important to tune the redundancy so as to match the link bandwidth while maximizing the expected performance. In this case, if a feedback about the channels is available, an easy tuning process that shapes the redundancy can be applied in order to match the network conditions by a simple trans-coder; these developments are left to future research.

Finally, in Fig. 4 the central and one description PSNR, for image Lenna encoded at $1 \mathrm{bps}$, are reported versus the total number of descriptions. Two values of inserted redundancy are considered, namely $\rho=0.05$ and $\rho=$ 0.225 . It can be noticed that for $\rho=0.05$ and high number of descriptions (i.e. more than 5 descriptions) the central performance impairment, due to the intrinsic MD redundancy devote for headers information and the extra inserted redundancy, is less than $1 \mathrm{~dB}$. On the other, hand decoding one description, when $\rho=0.05$, still guarantee acceptable quality despite the redundancy is minimal. We can notice that for more than 5 descriptions, and $\rho=0.05$, side quality is nearly constant. This is due to the high amount of missing information. An example of the visual quality obtained by decoding: a) one, b) two, c) three and d) four descriptions, where $N=4$ and $\rho=0.05$, is reported in Fig 5.

\section{CONCLUSIONS}

In this paper we proposed a practical MDC scheme for distributed storage of images. From the obtained results we conclude that it is possible to split the images among many servers with a minimal full-quality impairment while maintaining acceptable side performance, whereas the balanced descriptions characteristics means that all servers share the same level of importance and that all users have similar privilege. We also notice, that decoding one description still guarantee acceptable quality, even if the image is distributed over a large number of servers; this permits to have reliable system. The high flexibility of generating different number of descriptions, with tunable redundancy, makes it easy to match network characteristics, storage cost and quality of service requirements. Moreover, the inherent MDC scalability makes the proposed framework a good choice for heterogenous networks composed of users with different bandwidth. The possibility to decode any subset of descriptions allows to have fast access to a coarse quality image; successively the quality is improved by other descriptions. The generalization of the proposed algorithm to video storage system is left to future research.

\section{REFERENCES}

[1] V. Castelli, L. D. Bergman, I. Kontoyiannis, C.-S. Li, J. T. Robinson, and J. J. Turek, "Progressive search and retrieval in large image archives," IBM J. Res. Dev., vol. 42, no. 2, pp. 253-268, 1998.

[2] J. R. Smith, "Digital video libraries and the internet," IEEE Communications Magazine, Special issue on the Digital Library for Next Generation Internet.

[3] I. Richer and B.B. Fuller, "An overview of the magic project," M93B0000173, The MITRE Corp., Bedford, MA, (Available from http://www.magic.net.), Dec. 1993.

[4] S. Rhea, S. Wells, P. Eaton, D. Geels, B Zhao, H Weatherspoon, and J. Kubiatowicz, "Maintenance-free global data storage," IEEE internet computing, pp. 40-49, Oct. 2001.

[5] Q.M. Malluhi and W.E. Johnston, "Coding for high availability of a distributed-parallel storage system," IEEE Transactions on Parallel and Distributed Systems, vol. 9, no. 12, pp. 1237 - 1252, Dec. 1998.

[6] Yvan Leclerc, Martin Reddy, Lee Iverson, and Michael Eriksen, "The geoweb - A new paradigm for finding data on the web," 2001.

[7] M. Reddy, Y. Leclerc, L. Iverson, and N. Bletter, "Terravision ii: visualizing massive terrain databases in vrml," IEEE Computer Graphics and Applications, vol. 19, no. 2, pp. 30 - 38, Apr. 1999.

[8] D.A. Patterson, G. Gibson, and R.H. Katz, "A case for redundant arrays of inexpensive disks," in Proc. ACM SIGMOD, Chicago, IL, June 1988, pp. 109-116.

[9] V.K. Goyal, "Multiple description coding: compression meets the network," IEEE Signal Processing Magazine, vol. 18, no. 5, pp. 74-93, Sept. 2001.

[10] V. Vaishampayan, "Design of multiple description scalar quantizers," IEEE Trans. on Information Theory, vol. 39, no. 3, pp. 821-834, May 1993.

[11] E. Baccaglini, G. Barrenetxea, and B. Beferull-Lozano, "Performance of multiple description coding in sensor networks with finite buffers," in Proc. IEEE International Conference on Multimedia and Expo, July 2005, pp. 1460 $-1463$.

[12] T. Tillo and G. Olmo, "A novel multiple description coding scheme compatible with the jpeg 2000 decoder," IEEE Signal Processing Letter, vol. 11, no. 11, pp. 908-911, Nov. 2004.

[13] T. Tillo, E. Baccaglini, and G. Olmo, "A flexible multi-rate allocation scheme for balanced multiple description coding applications," in Proc. IEEE International Workshop on Multimedia Signal Processing (MMSP 2005), Nov. 2005.

[14] "Multiple description JPEG 2000 codec available at: www.telematica.polito.it/sas-ipl/," . 